Article

\title{
Interaction Tension: A Sociological Model of Attention and Emotion Demands in Video Gaming
}

\author{
Sebastian Deterding \\ Digital Creativity Labs, University of York, York, YO10 5GE, UK; E-Mail: sebastian.deterding@york.ac.uk
}

Submitted: 16 July 2019 | Accepted: 21 November 2019 | Published: 20 December 2019

\begin{abstract}
Video gaming actively demands players' attention, affording positive experiences like flow. Recent research has suggested to extend analysis from cognitive and physical to the social and emotional demands of gameplay. This article argues that Erving Goffman's concept of interaction tension offers a promising theoretical model for social demands. We report a re-analysis of qualitative interview data on the social norms of video gaming corroborating the model. As suggested by Goffman (1961) for gaming, video gaming features rich social norms regarding involvement. When spontaneously experienced and normatively demanded involvement misalign, players experience self-conscious disinvolvement and engage in unenjoyable, effortful self-control of their experienced and displayed involvement.
\end{abstract}

\section{Keywords}

gaming; Goffman; interaction tension; self-control; social demands; video games

\section{Issue}

This article is part of the issue "Video Games as Demanding Technologies" edited by Nicholas David Bowman (Texas Tech University, USA).

(C) 2019 by the author; licensee Cogitatio (Lisbon, Portugal). This article is licensed under a Creative Commons Attribution 4.0 International License (CC BY).

\section{Introduction}

Gaming, at least the desirable kind, binds our attention: We call it immersive, absorbing, engaging, or involving (Cairns, Cox, \& Nordin, 2014; Calleja, 2011). But how do games accomplish that? And why, in the age of the attention economy, do we so gladly part with this everscarcer resource?

Following flow theory, humans enjoy "order in consciousness" (Csikszentmihalyi, 1990, p. 39), yet consciousness naturally drifts toward disorder: Unmet desires, worries, aversions, and self-consciousness split and divert our attention (Csikszentmihalyi, 1990, p. 37). Hence, we seek out gaming and other "flow activities" because their "clearly structured demands...impose order" (Csikszentmihalyi, 1990, p. 58) and ensure that "attention is completely absorbed" (p. 53). Mood management theory similarly argues that people improve their mood by selecting media with a high intervention potential, the ability to absorb attention such that none is left to ruminate on negative thoughts (Reinecke, 2016)-and games have been found to provide just that (Bowman \& Tamborini, 2015).

\subsection{The Demands of Games and Challenging Media}

Past applications of flow and mood management theory to games have focused cognitive and physical demands on attention (Sweetser \& Wyeth, 2005), like solving puzzles or fast hand-eye coordination in jump-andrun games. Bowman's (2018a) recent “interactivity-asdemand" perspective suggests to extend this towards a more general theory. Games and interactive media, he argues, put cognitive and physical, but also emotional and social demands on users, which mediate their various experiential effects, not just attentive binding. This demand perspective is currently drawing academic attention, evident in a topical edited book (Bowman, 2018b) and the present thematic issue. It strongly overlaps with recent attempts to re-conceptualize challenge in games and media. Cole, Cairns, and Gillies (2015) for instance identified distinct functional versus emotional challenges in 
games, which map onto cognitive and emotional demand. Denisova, Guckelsberger, and Zendle (2017) distinguish cognitive, physical, and emotional game challenge, which map one-to-one to cognitive, emotional, and physical demands. Relatedly, eudaimonic media research suggests that audiences actively seek out "challenging media" for meaning and personal growth (Bartsch \& Hartmann, 2017). Here, challenge is conceptualized as "the extent to which users perceive they need to apply self-regulatory resources to control both cognitive and affective processes while processing media content" (Eden, Johnson, \& Hartmann, 2018, p. 355).

\subsection{Social Demands: From Separable Stimuli to the Constitutive Sociality of Gaming}

Arguably the most novel demand dimension proposed by Bowman (2018a) are social demands, "the extent to which a system triggers an implicit or explicit response in the user to the presence of other social actors." Under this heading, he comprises phenomena like social facilitation, presence, relatedness need satisfaction, but also selfpresentation and social norms and pressures. Bowman, Wasserman, and Banks (2018) subsequently developed and validated a video game demand scale, finding a discrete social demand factor comprising six items, which significantly correlated with relatedness need satisfaction. In the same volume, Peña (2018) presented a framework factoring out different social demands as "any component of the communicative process...that has an impact on the quantity and quality of the social ties of players and game audiences." In parallel, Bopp, Opwis, and Mekler (2018) identified "social challenges" as a subset of 'conventional' and emotional challenge in gameplay, comprising social conditions that produce a challenge (e.g., others ganging up or difficult team communication), managing other players' emotion, and genuine social emotions, chiefly pride in oneself and anger at others.

Useful as these accounts are, they present more of a first descriptive classification of related phenomena than theoretical models identifying distinct mechanisms that link these phenomena together: The 'social' of social demands variously refers to social antecedents (mediated or cued presence of other social actors in Bowman [2018a], Bowman et al. [2018]; social conditions in Bopp et al. [2018]) and social consequences (social ties in Peña [2018]; social emotions in Bopp et al. [2018]; social gratifications and need satisfaction in Bowman [2018a]). In addition, Peña (2018) and to some extent Bowman (2018a) present social demands as analytically separable from other forms of demand (e.g., Bowman [2018a, p. 15], "it is plausible that social demands can represent their own unique source"). In this, their metaphorical use of the word "demand" strangely elides its literal meaning: "An act of demanding or asking by virtue of right or authority" (Demand, n.d.). A demand is a communicative action drawing on a social norm to compel another person to act in a certain way.
Against this stand ethological, anthropological, sociological, and developmental play research (Burghardt, 2005; Henricks, 2015; Pellegrini, 2009) and sociomaterial accounts of video gaming (Stenros, 2015; Taylor, 2009) which argue that (video) gaming is always already social. 'Gaming' and 'games' are social categories constituted by shared meanings and actions, just like 'money' or 'marriage.' We need to be actively socialized into perceiving and realizing the affordances of game hardware and software (Hung, 2011). And what we do and feel during gaming is subject to a rich tapestry of specific norms. Playing any game requires us to enact these social orders, where 'gaming' or 'Pong' is a thing, where crossing this pixel line with that pixel dot is counted as 'scoring a point,' and where doing so is an appropriate reason to be cheerful (Sniderman, 1999). In other words, whatever a game object 'demands' of a player, the object and its demands are always already made out of matter as much as social norms, practices, and understandings, whose ongoing reproduction is mutually expected and sanctioned by the members of our society.

Is there a way to reconcile a demand perspective with this constitutive sociality of gaming? This article proposes that sociologist Erving Goffman's model of interaction tension provides just such a coherent theory of how constitutive social demands impact player experience. In brief, Goffman (1961) argues that when interacting with others, states of enjoyment, boredom, or (un)self-consciousness arise not just from the activity itself, but from the relative alignment of spontaneous and normatively demanded attentive and emotional involvement. In demand perspective terms, the social demands of games can be conceptualized as the social norms of 'proper' attentive-emotional involvement in gaming, with 'norm-fitting' as a socialpsychological mediator of player experience.

To illustrate the applicability of interaction tension to video gaming, after introducing the model, we here report a re-analysis of qualitative interviews with German adult players around social norms for attentive and emotional involvement in video gaming. Our data broadly corroborates but also qualifies Goffman's model in that misaligned spontaneous and demanded involvement is experientially characterized by effortful self-control more than boredom and self-consciousness, and that it can also be experienced during solitary gaming. We discuss wider ramifications for the demand perspective on video gaming as well as flow and mood management theory: Broadening the focus from game stimuli to social dynamics and contexts, including self-control as an important mediating process, and working out how social demands systemically structure the attention and emotion demands of game play.

\section{Theory: Interaction Tension}

Goffman's (1983) work centers on the interaction order, the specific social ordering that obtains when two or more people can immediately perceive and respond to 
each other's actions. Such response-present interaction uniquely affords joint attention, which enables the symbolic and finely coordinated action essential to human culture (Seeman, 2011).

To guide action and sense-making in responsepresent interaction, social groups develop a shared repertoire of frames, reoccurring types of situations such as 'going to the doctor' or 'lecture' (Goffman, 1986). Every frame entails roles that can (and have to) be taken on by actors and define what conduct is expected and appropriate for each role-taker. During socialization, children not only internalize frames and roles, but also become aware of and construct their self from others' responses, become emotionally invested in this self, and adopt the values of their group regarding desirable self traits: They learn to care about face, "an image of self delineated in terms of approved social attributes" (Goffman, 1967, p. 5). Social emotions like pride or embarrassment are the affective dimension of assessing how relevant others assess our self (Scheff, 2000; Turner \& Stets, 2006). They motivate us to act properly in response-present interaction, to fulfil role expectations, present a desirable self, show due regard to the other's self, and keep interaction flowing smoothly (Goffman, 1967, pp. 5-112).

By its nature, working response-present interaction requires mutual involvement. Goffman $(1963$, p. 36) defines involvement as "cognitive and affective engrossment" in an activity. As social animals, we have a natural propensity to get unselfconsciously involved in joint attentional foci. Such joint involvement mutually signals that the focal activity is worth and appropriate to unselfconsciously attend to (Goffman, 1967, pp. 113-167). Strong joint involvement fuels social cohesion and mobilization (Goffman, 1967, p. 113), as seen in e.g., raving sports fans watching a match together (Hatfield, Cacioppo, \& Rapson, 1994; von Scheve \& Salmela, 2014). Conversely, without joint involvement, response-present interaction quickly breaks down.

Therefore, as part of frames, social groups develop norms around "the structure of involvement in the situation" (Goffman, 1963, p. 193), who may legitimately open, join, leave, or close what kind of joint focal activity, and how deeply involved one may and ought to become in it. For most situations, the "expression of a particular allocation [of involvement] is obligatory" (Goffman, 1963, p. 37). Even worse, there is an "obligation of spontaneous involvement" (Goffman, 1967, p. 115). Take a party conversation: Not only are we expected to visibly attend and emotionally respond to the other-we also need to pull this off without it appearing belabored. Outwardly spontaneous involvement in conversation shows proper regard to the conversation partner, the norms of conversation, and maintains our face as a skilled conversationalist (Goffman, 1967, p. 114). Every type of situation asks for striking a different involvement depth: neither too distanced, nor too engrossed. Adults in particular are "obliged to express a margin of disinvolvement" (Goffman, 1967, p. 122).
And this leads us to interaction tension (Goffman, 1953, pp. 243-257, 1961, pp. 41-45). Whenever we interact with others, our spontaneous involvement aligns more or less well with the situation's normative expectations. If people's spontaneous involvement aligns with norms, people can allow themselves to stop reflexively monitoring and controlling their involvement (Goffman, 1986, p. 378). The resultant positive experience is "euphoric ease" (Goffman, 1961, p. 42). However, the sad normal state of affairs is "dysphoric tension," "some discrepancy between obligatory involvements and spontaneous ones" (Goffman, 1961, p. 44). This leads participants to experience "alienation from interaction" (Goffman, 1967, p. 113): They feel bored, awkward, uneasy. They have to effortfully fabricate an involvement display that fits the situation against their inclinations, and they are likely to become self-conscious about this fabricated face, the thoughts and feelings of the other participants, and whether the situation is 'working.' This alienation is just as contagious as engrossment (Goffman, 1967, pp. 125-129).

Now just like flow theory suggests that the structure of gaming activity affords flow, Goffman (1961, p. 43) argues that in gaming, "euphoric interaction is relatively often achieved: gaming is often fun." This is because games are purpose-designed to spontaneously excite and bind deep joint involvement, and the situational norms of gaming allow and demand just such deep joint involvement. As a result, the existence of involvement norms disappears from participants' awareness. The paradox of gameplay is that it is "shared, obligatory, spontaneous involvement" (Goffman, 1961, p. 43).

In summary, Goffman provides an integrated model of the social-psychological dynamics of social norms, attention, and emotion in gameplay: When co-present participants' involvement is spontaneously excited and bound by the situation's sanctioned focal activity, they reproduce and amplify each other's involvement and experience positive unselfconscious ease. When their spontaneous involvement mismatches situational obligations, they experience dysphoric tension and become alienated from interaction (drifting with their attention, bored, self-conscious), which is likewise contagious, while participants try to overtly display spontaneous involvement in the legitimate focal activity.

\section{Method}

Clear as this model may be, it was developed from field observations of mid-20th century Shetland Island communities and US casinos. To explore its fit with contemporary video gaming, we conducted a focal re-analysis of a larger data set of interviews with German adult video game players on the social norms of gaming (reported in Deterding, 2014).

Following Maxwell (2004), the original study developed an initial conceptual framework to structure data collection and analysis. Specifically, it started from key 
dimensions of situational norms as theorized in frame analysis (Goffman, 1986): settings, objects, roles, internal organization, metacommunication, attention, emotion, rules for action and communication, and situational boundaries. Because social norms are typically taken-forgranted and therefore hard to elicit, the original study used semi-structured episodic interviews (Flick, 1997) to elicit a broad range of 'critical incidents' where participants remembered a norm violation, which typically makes norms consciously available. We then probed participants to expand on the violated norm. Second, inspired by the grounded theory principle of constant comparison (Corbin \& Strauss, 2008), the study intentionally recruited participants who engaged in both 'canonical' leisurely gameplay and 'atypical' gameplay-as-work performed by game journalists, game designers, game researchers, and esports athletes. Participants were asked to recall 'typical' kinds of leisurely gameplay situations they engage in, to then compare these to the counterpart in non-leisurely work-as-play, thus foregrounding norm differences. To ensure all our concepts were grounded in data, the study followed the grounded theory principles of constant comparison and theoretical sampling (Corbin \& Strauss, 2008): It gathered and coded data in parallel, comparing each new datum against existing concepts, revising, dropping, or adding concepts as required by the data, adapting interview script and participants based on emerging questions until we reached theoretical saturation.

All interviews were conducted in German with adult native German-speaking participants across Germany between March 2011 and May 2012. Participants were purposely sampled for diverse backgrounds in age, gender, experience across game genres, devices, and social contextures. We conducted 19 interviews (about 1,900 minutes total recordings) until we reached saturation, in line with prior findings (Guest, Bunce, \& Johnson, 2006). We transcribed recordings together with field notes and coded them using the qualitative data analysis software MAXQDA. This produced 3,241 coded segments across 181 initial codes, which resulted in 24 focused codes, 6 axial categories and the central theme of "gaming modes." This theme captured that we found no single uniform set of norms for video gaming, but rather five different sub-types of leisurely gaming and four different sub-types of gaming-as-work, each with their own internally coherent set of norms that would support the sub-type's purpose. E.g., we found a socializing gaming mode whose norms where structured around experiencing social connection, or a competitive mode around achievement display before others, or an esports training mode structured around improving skill. As will become apparent, involvement norms similarly varied with gaming modes.

For the present article, we re-analyzed the data set described above. Since Goffman (1961) identified involvement as emotional and attentive engagement resulting in unselfconscious engrossment, we started re- analysis from 447 passages coded for 'emotion norms,' 'attention norms,' and 'tension/flow.' 'Attention norms' and 'emotion norms' were predefined focused codes that structured data generation, collection, and analysis, and proved analytically distinct throughout the iterative collection-coding-analysis process. The code 'tension/flow' emerged from the data as 'experiences of selfcontrol and self-awareness,' which we only later, during write-up, formally connected to Goffman's terms because of their mutual fit, inviting additional coding cycles sensitized by the interaction tension model. As such, the present study constitutes an instance of "double-fitting" (Baldamus, 1972) between empirical material analysis and conceptual framework, in which iteratively switching between the two mutually refines and differentiates each one-a qualitative analysis approach characteristic for Goffman's own work (Williams, 1988).

Interview transcripts and field notes in the German original as well as the code tree of the present study are available at https://osf.io/3w4js. All data quoted below is translated from the German original.

\section{Results}

\subsection{Involvement Norms}

\subsubsection{Mandatory Spontaneous Involvement}

Congruent with Goffman, we found a rich landscape of norms regulating video gaming involvement. Maybe the most basic norm of leisurely gaming voiced by participants is that one ought to spontaneously want to play:

Interviewer: Is there something you shouldn't do during playing?

P1: I don't know, if you want to do something else, then you should do something else. And if you want to play video games, then you should play a video game.

Enjoyment was seen as the legitimate official purpose of leisurely gaming. While this was voiced as a mere expectation in single-player gaming, it turns into a positive normative demand in multi-player gaming, as satisfying gaming was seen to depend on the others mustering some minimum visible involvement: "People of course also have to be there with a minimum stake, in wanting and tactics...because otherwise the game collapses" (P3).

\subsubsection{Social Interaction of Involvement}

Four ways personal involvement affected the involvement of others emerged. First, distracted players tend to break the flow of gameplay and create boring pauses by missing their turn. Second, their inattentiveness leads them to play sub-optimally, making either for a poor teammate or boring opponent, as the following passage nicely illustrates: 
P7: We played soccer, so we played Pro Evolution Soccer 2012, with several friends, online, against each other. So we were a two-player team in our living room, and in Vienna friends of us were sitting, and we played against them. And the one did indeed look on his mobile phone from time to time, and then I told him: 'Hey, let that be! I don't want to lose here, against them.' You're quite captivated there, and-it was similar when I once, with my friends, every two weeks we play, we make a gaming night and play Golf together, Tiger Woods. And it's annoying when people don't press 'continue.' That is, when they, like, talk with each other or want to go have a smoke or something like that, so that's, that impedes the game flow, simply because you don't get further.

Participants reported social contagion as a third way others' involvement affected their own. As one put it, in solitary StarCraft play, they would just 'breathe off' excitation, whereas in playing seated together with a teammate, expressing shared emotion would intensify it:

P10: When I play StarCraft alone at home and win, then I also say 'Puh' [exhales]. But that way [playing with their friend], you also work each other up a little. That is because you, like, communicate so much with each other and...also curse verbally somehow about the enemy...and then after a, after a win, then you also make a high five. So there the experience is something totally different.

Others' verbal or bodily display of emotional involvement amplifies and validates one's own. Says an esports athlete:

P15: Especially when you play on LAN...you rejoice more, because you see your teammates, you can, when he [got] something important, you can shake him and say, like, 'Great, man!' But it's just like that when you see your team lose....Then all five [of the team] sometimes sit like [makes depressed face] and are just upset. And yes, the emotions are a bit higher.

Conversely, displaying continuous negative affect was seen to 'infect' others' mood. Asked whether they remembered an "inappropriate" way of playing, P2 named "people who are simply in the mood for nothing. Who document that [laughs] then, from 'Ahhh, that's stupid' or, when it's their turn, somehow are frustrated the whole time."

Fourth and finally, participants in a known or visibly bad mood made co-present others conscious about their lacking enjoyment, which led co-present others to inhibit their usual behavior. As P15 explains, "when a player from my team...had just broken up with his girlfriend, then [he is] a little down....When you notice that, [you] shouldn't taunt him on top of that."

\subsubsection{Involvement Display}

As individual involvement depends on that of the others, participants voiced a normative expectation to not just experience but also display fitting involvement: "One should show fun. So if you don't connect to the game and don't enjoy it, one should still somehow show that it was a nice situation to interact with friends" (P9).

These required displays were especially pronounced around wins and losses:

P2: Yes, so in a group game, in a group game it is expected that you show elation when you have achieved something, somehow....You should certainly also be appropriately frustrated when something doesn't work, and not say: 'Ahh, who cares.' And then...in group situations like with the Kinect, there it's certainly also the case that you should appropriately be happy for somebody else, if somebody made a new high score, because that's certainly socially, like, desired.

Besides 'working up' appropriate involvement, participants also reported actively inhibiting felt emotions in order to save face in the situation. As P5 noted on playing multiplayer online shooters:

P5: So when I'm continually killed by the same player, then you effectively develop such a kind of hatred...that's effectively also an admission that you are inferior [pause]. But that I wouldn't say [openly].

Interviewer: What kind of reaction do you show instead?

P5: Nothing....There I completely contain myself. Because that, that is, as I said, such a matter of honor.

That said, the social norms of leisurely gaming typically allow for more intense emotion expressions than everyday conversation, because the gaming frame earmarks them as 'non-serious,' not targeted at the actual biographical person of the other. Says one participant:

P4: Anger, aggression, when you're playing video games together...those are all things accompanying gameplay, that are often also playful.....Nothing that is wrong or so.

Interviewer: [D]o you remember a situation where someone took that the wrong way?

P4: No, no, no. That's with the people with whom I've played up to now, so that they [pause] take that in a way that shows me [that] they see that similarly. Evaluate that in the same way, are apparently [pause] socialized similarly, know that that's part of gaming and not meant in a malign manner, is even part of the whole. 
In sum, participants described striking a precarious balance of disinvolved involvement-trying to win but not 'too much,' visibly celebrating wins and bemoaning losses, and yet not 'really' minding either. To fail at this balance is to count as a "sore winner" or "sore loser." Either of which is problematic because it again spoils the others' involvement, as this participant explains:

P17: No, you are a sore loser if you, if you burden the others with your own frustration. That means, when you vent your anger...and you're in a sour mood and that becomes a burden for the others.... mean, that you get angry when you lose, that's alright. That's normal....But when you then become annoying for the others...then, then I find that a sore loser.

\subsection{Attention Norms}

\subsubsection{Attentive Access}

From his field studies of casino gambling, Goffman (1986, pp. 133-136) noted that players and bystanders are expected to limit their attentive access to certain legitimate information streams and not access certain other, legitimately 'hidden' streams, such as the hand of another Poker player. These attentive access norms were supported by material arrangements like removing mirrors from the walls. We found similar norms and practices in video gaming. As one interviewee described a multiplayer LAN party:

P5: Yes. Looking on the screen is taboo.

Interviewer: Ha. [That means?]

P5: [Yes, naturally] I mean, not, not on your own screen, that's obvious [laughs]. So of course you may look on your own screen, but the tables are placed in a way...that the screens always stood with their backs to each other. So that you really only saw your friend and could not look on the screen of the other person.

\subsubsection{Attentive Focus and Depth}

While participants concurred that some spontaneous involvement was expected in any leisurely gaming, norms starkly differed when it came to the expected focus and depth of attention: what to attend to, and what forms of distraction or interruption would be acceptable. These norms seemed to functionally align with the legitimate purpose of the situation. For instance, they differed with the degree to which the game played would require undivided attention. In a turn-based social game like FarmVille, interruptions and distractions didn't matter:

Interviewer: [I]S it okay to be interrupted with FarmVille?
P12: Yes, most definitely. 'Cause there's nothing that necessarily happens during that.

Compare this with a competitive real-time multi-player game like StarCraft:

Interviewer: How would you have told whether one of you was distracted or not?

P10: Well, [pause] actually already just somehow looking away from the screen...turning my head to you and talk. That would actually already be distraction...because then for perhaps five seconds or so I don't see what's happening there. What under certain circumstances can already be decisive in the game.

Normatively expected focus and depth of attention also varied with gaming mode (Deterding, 2014). In gaming modes valuing player peak performancehardcore, competitive, and esports tournament gaming-participants reported strong and strongly enforced expectations to maintain intense unfailing attentive focus on gameplay via the legitimate interface. In social gaming, by contrast, such deep, exclusive gameplay focus was seen as inappropriate: Here, gameplay is a means for social connection. Hence, participants are expected to maintain attention of the others' current emotional states and engage in the legitimate main involvement of conversation:

Interviewer: Is there something you have to do during party gaming to play 'correctly'?

P9: I believe [it is] to have enough of a distance from the game and you're still aware in what context the whole thing takes place, namely in an amicable frame, in, in a party frame. To be jolly and not too focused and fully focus on it and no communication happens, then it really failed, I would say. Because it's really about getting to interact with each other, having fun, communicating, being able to laugh about it, being able to laugh about yourself. I would say, if somebody would fully shut himself off and focuses exclusively on it and doesn't interact with co-players, then I would say it failed, yes.

Interviewer: Focus on what [exactly]?

P9: [On the] game, on the action. If somebody is completely in his own world and fully shut off and takes it too over-ambitiously, I would say. Yes. I would say, the way I play Battlefield, I really wouldn't be fit for social contact [laughs]. Yes. 


\subsection{Conditions of Interaction Tension}

\subsubsection{Response-Present Others}

Participants uniformly reported that response-present others would make involvement norms salient and lead them to manage their involvement display. Conversely, being alone led them to not manage their displayed involvement:

P9: So if I feel unobserved, in my private rooms, then I can show any emotion, because there would be nothing inappropriate in doing so, because I wouldn't offend anyone with it. At most I would offend myself [laughs].

This was voiced as one reason why multiplayer online games would sometimes invite disinhibited display: The game would not transmit bodily emotion display, and even if players were to express e.g., anger over voice or text chat, anonymity would save their face:

P19: So there [in online games] I let my emotions run free. You can do that there, because you don't speak to the other. So you can't directly hurt them. So you also drop curse words...you would never say in the face of a colleague.

\subsubsection{Gaming versus Public Frames and Audiences}

Notably, response-present others evoked different norms depending on the type of frame and audience. It is largely taken for granted but significant that most leisurely gaming takes place within physically shielded private places like a living room, either alone or surrounded by co-players and an audience who are all aware of and have at least implicitly assented to the fact that the current situation is video gaming. This sets and licenses joint expectations around gaming-typical involvement and makes displayed behavior intelligible. As one player noted, when he played StarCraft online on his laptop communicating with his team members via Voice over Internet Protocol in his mother's kitchen, he would feel embarrassment when his mother entered the room, who did not know the game:

P10: If somebody, somebody who has now clue about, no view of the game and hears me talking. So that's somehow awkward for me, because somebody who doesn't know what I'm doing just hears these weird, cryptic communicative lumps of language from me.

This issue becomes even more salient in non-gaming encounters. In "public traffic" like bus stops, markets, or trams, norms demand adults to pay and draw minimal overt attention (Goffman, 1963). In public, players are exposed to an unknown audience that may not understand nor approve of 'gaming.' Hence, participants stated that they would strongly inhibit their involvement displays in public:

Interviewer: If you play a mobile game...in comparison to playing at home alone...is there a difference in what emotions you can or are allowed to express?

P7: Since I am then mostly in a public surrounding, loud screaming or throwing that thing in the corner are not an option. Although you would really want to do it, you have to restrain yourself a bit there and, let's put it this way, appear a bit more suited for public.

Similarly, a participant reported that during her work hours as a game designer at the office, although playing a game was part of her official professional duties, she would fit her emotion display to the office frame:

P9: Because I am sitting with two other colleagues in the office. And there, a certain behavior codex is desired. I don't want my colleagues to [see me] curse loudly or bang my hand on the table [which she would do playing at home]. A certain body posture is simply proper. So I can't just let myself slump in the chair in front of the computer, as it sometimes happens at home, when I relax. Instead I'm sitting upright and straight and really try [laughing] to make an interested impression. And yes, that's a different composure toward the game.

\subsubsection{Solitary Dysphoric Tension}

Interestingly, our data showed that involvement norms could also become salient and cause dysphoric tension during solitary play. We mainly observed this during work-at-play. Participants would experience a spontaneous pull to let themselves become unselfconsciously involved in gaming, but their current work frame made salient that they ought to engage with the game in a disengaged, analytic fashion, e.g., to analyze it as a game designer or review it as a game journalist. As one game journalist described his experience:

P1: So I really think that [pause] that when I'm playing reviewingly...that I am somehow taking part cognitively in a different way. That means, beforehand I'm already in this mood: 'Okay, I do, I work now, and I try to grasp intellectually what is going on here now.' And in a normal non-reviewing gaming situation exactly that is a great advantage for me, that I don't try to grasp things intellectually, but instead let myself be drifted by the sensual impressions, and that can of course emotionally evoke very different things, yes? For instance, at the end of Metal Gear Solid 4 I cried like a baby, because it was a completely different situation than for Peter, who, he wasn't yet with the game magazine then, but he reviewed the game back then. [O]f course I have to capture these emo- 
tions, but I can't give myself so fully into them that over that I forget to bring that into words.

\subsection{Experiences of Interaction Tension}

The previous quote nicely highlights the major experiential quality participants reported with regard to interaction tension. Goffman (1961) suggested that high or dysphoric interaction tension was characterized by attentive drift, boredom, and awkward self-consciousness, and low tension or euphoric ease by unselfconscious attentive and emotional absorption. Interestingly, our data showed little awkward self-consciousness associated with dysphoric tension. Boredom was more frequently reported, when social or professional norms demanded that one continued to play despite a lack of spontaneous interest: "The thing that you play with people in coop [cooperative mode], and you don't want to anymore, and another person still wants to" (P3). Far more salient for dysphoric tension was the experience of unpleasant, effortful self-control, be it to force continued attention on the activity, inhibit emotional responses, or maintain analytic distance. Playing for work, for instance, a game journalist stated that "[I] must not let myself drift" (P1), emotions "are more inhibited, so...the game experience is markedly more inhibited at the office, because I cannot let myself go" ( $\mathrm{P} 9$, a game designer), and "if that is necessary, that I play every day, even if I don't want to, then I force myself to do it" (P13, esports athlete). All this resulted in an overall more muted, distanced, and less emotionally intense experience, "less ambitious, more passive" (P9).

Descriptions of euphoric ease interestingly align strongly with flow theory, down to the very words people use for it:

P3: You notice it when you don't notice anything anymore... when the state of unselfconsciousness sets in and you get into this flow...certain emotions [pause] come up in...a more pure form. That is, when you let yourself fall or something, and simply savor that, then it may be that you...certain things are simply more intense.

"Letting yourself go" or "letting yourself fall" were frequent turns of phrase for moments of low interaction tension, especially in solitary gaming, indicating that again, self-control is a crucial experiential dimension of interaction tension.

\section{Discussions and Conclusion}

\subsection{Main Findings}

Our data broadly supports Goffman's interaction tension model for contemporary video gaming. First, we indeed found a nuanced set of social involvement norms. In leisurely gaming, gameplay ought to be spontaneously involving, and players ought to display a delicate balance of disinvolved involvement to optimally support mutual involvement: showing investment in winning to make the others' investment feel justified, yet not becoming so over-involved as to overlook the other's feelings or burden them with one's frustration. Players ought to limit their attention to legitimate information channels. And they ought to maintain an attentive focus and depth matching the functional requirements of the game and the situation's official purpose (e.g., socializing versus competing). Thus, we found that involvement norms are modulated by gaming modes, games, audiences, and settings, which Goffman did not suggest. Also, while response-present others made involvement norms salient, people reported dysphoric tension even in solitary play, namely when internalized professional norms demanded analytic detachment while the game afforded unselfconscious engrossment.

Second, we observed several social involvement dynamics. Yet where Goffman only identified selfconsciousness dynamics, we observed additional dynamics of emotion contagion through emotion display, and distraction leading players to create undesired breaks in gameplay, or playing suboptimally.

Third and finally, as suggested by Goffman, we found that low interaction tension was experienced positively, and high tension negatively. Here, our data qualifies Goffman's claim that dysphoric tension chiefly revolves around boredom and self-consciousness: For our participants, effortful self-control of attention and emotion and a consequential dampening of emotional intensity was far more pronounced and negatively valued. Meanwhile, their reports of euphoric ease aligned closely with flow states, which brings us back to the beginning.

\subsection{Contextualisation}

We opened this article with the question of how video games absorb our attention and why we find such absorption desirable. Flow theory answers that cognitively and physically demanding activities absorb attention, which affords positively valenced order in consciousness and blocks attention-wandering to negatively valenced worries and self-conscious concerns (Csikszentmihalyi, 1990). Bowman (2018b) and others have suggested extending such analyses from physical and cognitive to emotional and social demands. So, what does interaction tension, as we found it in contemporary video gaming, add to this debate?

For one, it contributes a genuine social-psychological construct for social demands. Following Goffman, we can specify one kind of social demands of gaming as social involvement demands or the norms for 'appropriate' gaming involvement. There are certainly many other kinds of social demands in gameplay, but these appear conceptually and mechanistically distinct.

Notably, second, this kind of social demand is not a separable 'add-on' to cognitive (including attentive) 
and emotional demands, as suggested by Peña (2018) or Bowman (2018a): they pre-structure and co-constitute them. There are social demands on attention (cognition) and emotion, in presence and absence alike: The absence of salient social demands in solitary play is just as impactful as their presence in multiplayer encounters. Furthermore, social demands are not exhausted by what is triggered by a game or even other co-present players: They also always already arise from internalized norms about gaming and the gaming mode in question, as instances of interaction tension in solitary professional play demonstrated. Put differently, the emotional and attentive demands of video games arise at the intersection of material features, subjective dispositions, and social contextures (Elson, Breuer, \& Quandt, 2014). Interaction tension captures exactly this kind of higher-level dynamic or relational phenomenon.

It concurs with flow and mood management theory that activities that spontaneously afford involvement block worry and self-consciousness, but adds a crucial social-psychological loop: To be flow-affording or intervening, activities need to align not just with players' subjective dispositions (e.g., matching game challenge and player skill), but also with the social norms of the surrounding situation. Misalignment not only affords boredom or self-consciousness: Often, social norms will compel players to engage in effortful self-control (Baumeister, Vohs, \& Tice, 2007) to align overt behavior with norms, and that is negatively valenced. Bopp et al. (2018) found that players sometimes needed to actively self-regulate intense negative emotions to 'function' in-game or outof-game. Eudaimonic media research posits that people sometimes willingly self-control to engage with demanding media that promise personal growth (Eden et al., 2018). Our findings suggest that media-related selfcontrol is not just a practical matter of functioning or a price worth paying for personal growth: People dislike the strain of self-control involved in fitting displayed involvement into social norms. Therefore, they actively seek out or create media reception situations in which spontaneously afforded and socially demanded involvement align, such as watching a 'tearjerker' home alone or in the anonymity of a darkened movie theatre, where crying in public is both accepted and unnoticed.

\subsection{Limitations}

The qualitative nature and small, culturally, and temporally homogeneous sample of the present study limits its generalizability. We also note that the present article reports a re-analysis of qualitative data from an original study with a different focal theme (Deterding, 2014). While said original study stopped data collection on reaching theoretical saturation, the codes and themes of the present study are not necessarily fully theoretically saturated.

\subsection{Outlook}

A next step is to perform quantitative studies across divergent contexts to test the relations proposed in interaction tension. Should these bear out, interaction tension is arguably only the first opening of the socialpsychological dynamics of gameplay. For instance, the model fits and identifies a potential systematic place for other important social-psychological phenomena and mechanisms like social emotions (Turner \& Stets, 2006), joint attention (Seeman, 2011), emotional contagion (Hatfield et al., 1994), or collective emotions (von Scheve \& Salmela, 2014) within video gaming. The literature on these phenomena specifies, but also qualifies and at times goes beyond the relations captured by Goffmanjust as the social norms of gaming include but exceed involvement and its display.

\section{Acknowledgments}

This work was conducted in the Digital Creativity Labs (digitalcreativity.ac.uk), jointly funded by EPSRC/ AHRC/InnovateUK under grant No. EP/M023265/1.

\section{Conflict of Interests}

The author declares no conflict of interests.

\section{References}

Baldamus, W. (1972). The role of discoveries in social science. In T. Shanin (Ed.), The rules of the game: Crossdisciplinary essays on models in scholarly thought (pp. 276-302). London: Tavistock Publications.

Bartsch, A., \& Hartmann, T. (2017). The role of cognitive and affective challenge in entertainment experience. Communication Research, 44(1), 29-53. https://doi. org/10.1177/0093650214565921

Baumeister, R. F., Vohs, K. D., \& Tice, D. M. (2007). The strength model of self-control. Current Directions in Psychological Science, 16(6), 351-355.

Bopp, J. A., Opwis, K., \& Mekler, E. D. (2018). “An odd kind of pleasure": Differentiating emotional challenge in digital games. In Proceedings of the 2018 $\mathrm{CHI}$ Conference on Human Factors in Computing Systems. New York, NY: ACM. https://doi.org/10.1145/ 3173574.3173615

Bowman, N. D. (2018a). The demanding nature of video game play. In N. Bowman (Ed.), Video games: $A$ medium that demands our attention (pp. 1-23). New York, NY: Routledge.

Bowman, N. D. (Ed.). (2018b). Video games: A medium that demands our attention. New York, NY: Routledge.

Bowman, N. D., \& Tamborini, R. (2015). "In the mood to game": Selective exposure and mood management processes in computer game play. New Media \& Society, 17(3), 375-374. https://doi.org/10.1177/ 
1461444813504274

Bowman, N. D., Wasserman, J., \& Banks, J. (2018). Development of the video game demand scale. In N. Bowman (Ed.), Video games: A medium that demands our attention (pp. 207-232). New York, NY: Routledge.

Burghardt, G. M. (2005). The genesis of animal play: Testing the limits. Cambridge, MA: MIT Press.

Cairns, P., Cox, A., \& Nordin, A. I. (2014). Immersion in digital games: Review of gaming experience research. In M. C. Angelides \& H. Agius (Eds.), Handbook of digital games (pp. 337-361). Hoboken, NJ: John Wiley \& Sons.

Calleja, G. (2011). In-game: From immersion to incorporation. Cambridge, MA, and London: MIT Press.

Cole, T., Cairns, P., \& Gillies, M. (2015). Emotional and functional challenge in core and avant-garde games. In Companian Proceedings of the 2015 Annual Symposium on Computer-Human Interaction in Play (pp. 121-126). New York, NY: ACM. https://doi.org/10. 1145/2793107.2793147

Corbin, J., \& Strauss, A. L. (2008). Basics of qualitative research: Techniques and procedures for developing grounded theory (3rd ed.). Los Angeles, CA, London, New Delhi, and Singapore: Sage.

Csikszentmihalyi, M. (1990). Flow: The psychology of optimal experience. New York, NY: Harper and Row.

Demand. (n.d.). In Oxford English Dictionary. Retrieved from https://www.oed.com/view/Entry/49580

Denisova, A., Guckelsberger, C., \& Zendle, D. (2017). Challenge in digital games: Towards developing a measurement tool. In Proceedings of the $2017 \mathrm{CHI}$ Conference Extended Abstracts on Human Factors in Computing Systems (pp. 2511-2519). New York, NY: ACM. https://doi.org/10.1145/3027063.3053209

Deterding, S. (2014). Modes of play: A frame analytic account of video game play (Unpublished Doctoral dissertation). Hamburg University, Hamburg, Germany. Retrieved from http://ediss.sub.uni-hamburg. de/volltexte/2014/6863

Eden, A., Johnson, B. K., \& Hartmann, T. (2018). Entertainment as a creature comfort: Self-control and selection of challenging media. Media Psychology, 21(3), 352-376. https://doi.org/10.1080/15213269. 2017.1345640

Elson, M., Breuer, J., \& Quandt, T. (2014). Know thy player: An integrated model of player experience for digital games research. In M. C. Angelides \& H. Agius (Eds.), Handbook of digital games (pp. 362-387). Hoboken, NJ: John Wiley \& Sons.

Flick, U. (1997). The episodic interview: Small-scale narratives as approach to relevant experiences. London: LSE Methodology Institute.

Goffman, E. (1953). Communication conduct in an island community. Chicago, IL: University of Chicago.

Goffman, E. (1961). Encounters: Two studies in the sociology of interaction. Indianapolis, IN: Bobbs-Merrill.

Goffman, E. (1963). Behavior in public places: Notes on the social organization of gatherings. New York, NY:
The Free Press.

Goffman, E. (1967). Interaction ritual: Essays on face-toface behavior. New York, NY: Pantheon Books.

Goffman, E. (1983). The interaction order. American Sociological Review, 48(1), 1-17.

Goffman, E. (1986). Frame analysis: An essay on the organization of experience. Boston, MA: Northeastern University Press.

Guest, G., Bunce, A., \& Johnson, L. (2006). How many interviews are enough? An experiment with data saturation and variability. Field Methods, 18(1), 59-82. https://doi.org/10.1177/1525822X05279903

Hatfield, E., Cacioppo, J. T., \& Rapson, R. L. (1994). Emotional contagion. Cambridge: Cambridge University Press.

Henricks, T. S. (2015). Play and the human condition. Champaign, IL: University of Illinois Press.

Hung, A. C. Y. (2011). The work of play: Meaning-making in video games. New York, NY: Peter Lang.

Maxwell, J. A. (2004). Qualitative research design: An interactive approach (2nd ed.). Thousand Oaks, CA: Sage.

Pellegrini, A. D. (2009). The role of play in human development. New York, NY: Oxford University Press.

Peña, J. (2018). A communication model of the social demands in video games. In N. Bowman (Ed.), Video games: A medium that demands our attention (pp. 125-144). New York, NY: Routledge.

Reinecke, L. (2016). Mood management theory. In P. Rössler, C. A. Hoffner, \& L. van Zoonen (Eds.), The international encyclopedia of media effects (pp. 1-13). Hoboken, NJ: John Wiley \& Sons. https://doi.org/10. 1002/9781118783764.wbieme0085

Scheff, T. J. (2000). Shame and the social bond: A sociological theory. Sociological Theory, 18(1), 84-99.

Seeman, A. (Ed.). (2011). Joint attention: New developments in psychology, philosophy of mind, and social neuroscience. Cambridge, MA: MIT Press.

Sniderman, S. (1999). Unwritten rules. The Life of Games. Retrieved from http://www.gamepuzzles.com/tlog/ tlog2.htm

Stenros, J. (2015). Playfulness, play, and games: A constructionist ludology approach. Tampere: Tampere University Press.

Sweetser, P., \& Wyeth, P. (2005). GameFlow: A model for evaluating player enjoyment in games. Computers in Entertainment, 3(3). https://doi.org/10.1145/ 1077246.1077253

Taylor, T. L. (2009). The assemblage of play. Games and Culture, 4(4), 331-339. https://doi.org/10.1177/ 1555412009343576

Turner, J. H., \& Stets, J. E. (2006). Sociological theories of human emotions. Annual Review of Sociology, 32(1), 25-52. https://doi.org/10.1146/annurev. soc.32.061604.123130

von Scheve, C., \& Salmela, M. (Eds.). (2014). Collective emotions. Oxford: Oxford University Press.

Williams, R. (1988). Understanding Goffman's methods. 
In P. Drew \& A. Wootton (Eds.), Erving Goffman: Exand Oxford: Polity Press.

ploring the interaction order (pp. 64-88). Cambridge

\section{About the Author}

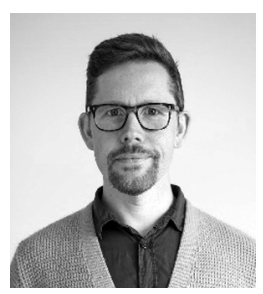

Sebastian Deterding is a Researcher/Designer working on wellbeing-driven experience design. He is a Reader at the Digital Creativity Labs at the University of York, Founder and Director of the design agency Coding Conduct, and Co-Editor of The Gameful World: Approaches, Issues, Applications (MIT Press, 2015), and Role-Playing Game Studies: Transmedia Foundations (Routledge, 2018). 